\title{
Eclipse Mapping the Flickering in HT Cas
}

\author{
William F. Welsh, J.H. Wood \\ Keele University, Dept. of Physics, Keele, Staffordshire ST5 5BG, UK
}

\section{Introduction}

Flickering is a well-known, though poorly understood, signature of accretion in cataclysmic variables. HT Cas is an excellent system in which to study flickering because its well determined characteristics allow a calibration of the experimental system (see Horne et al. 1991).

Patterson (1981) argues that the flickering in HT Cas originates from regions very near the white dwarf. In U Gem, the flickering appears to be associated with the bright spot (Warner \& Nather 1971). In RW Tri, the flickering occurs throughout the disk but is centrally concentrated (Horne \& Stiening 1985); the same holds true for V2051 Oph (Warner \& Cropper 1983).

HT Cas is a $16^{\mathrm{m}} \cdot 4$ eclipsing dwarf nova with a binary period of $106 \mathrm{~min}$ and an inclination of $\sim 81^{\circ}$. The entire eclipse last about $15 \mathrm{~min}$ with the white dwarf totally eclipsed for $\sim 5 \mathrm{~min}$. The mass ratio is 0.15 with the white dwarf having mass $\sim 0.6 M_{\odot}$ (Horne et al. 1991).

We obtained 20 eclipses of HT Cas between 1982 and 1991 in $U, B$ and $V$ at Mt. Palomar and also McDonald Observatory with $0.5-2 \mathrm{sec}$ integrations. These data were folded on the ephemeris given by Horne et al. (1991) and corrected for the $30 \mathrm{sec}$ drift in the phasing as noted by Wood et al. (1995).

\section{The flickering light curve}

The mid-eclipse flux in each of the 20 eclipses is not all the same. This is most likely due to long-term secular trends. To remove this contamination we applied a small additive constant to each light curve so that the mean flux at mid-eclipse remained constant.

The twenty eclipse light curves were combined to form a mean light curve. Deviations from the mean light curve were then recorded and then the rms scatter computed at each orbital phase. Note that the rms has been debiased to remove the expected contribution from photon noise.

The rms at each orbital phase gives us an "rms light curve", which is essentially a measure of the amplitude of the flickering versus time. This rms or 
flickering light curve was then flattened by dividing by a spline so that the mean flux remained constant outside of eclipse.

\section{Eclipse mapping}

The eclipse mapping technique developed by Horne (1985) has been applied to the flickering light curves. Using the maximum entropy method, the eclipse mapping technique produces an image of the accretion disk that is an optimum solution to two conflicting constraints: (1) the predicted light curve must fit the data ( $\chi^{2}$ constraint), (2) the image must contain as little structure as possible (the maximum entropy constraint). In addition, the maps are further constrained to be as azimuthally smooth as possible.

\section{Conclusions}

For HT Cas, we have the following preliminary results:

- The color of the flickering is blue.

- The eclipse of the flickering region is total in the $U$ band, and probably also in the $B$ and $V$ bands.

- The bright spot at the edge of the disk produces negligible flickering.

- Though concentrated towards the center, the flickering is not confined to the innermost region of the disk.

- The flickering brightness temperature vs. radius is more steep than the mean disk (see Wood et al. 1992), i.e., the relative amount of flickering increases toward the center of the disk.

- The brightness temperature vs. radius of the flickering is too flat for an optically thick accretion disk model.

\section{References}

Horne K., 1985, MNRAS 213, 129

Horne K., Stiening R., 1985, MNRAS 216, 933.

Horne K., Wood J.H., Stiening R., 1991, ApJ 378, 271.

Patterson J., 1981, ApJS 45, 517.

Warner B., Nather R., 1971, MNRAS 152, 219.

Warner B., Cropper M. 1983, MNRAS 203, 909.

Wood J.H., Horne K., Vennes S., 1992, ApJ 385, 294.

Wood J.H., Naylor T., Hassall B.J.M., Ramseyer T.F., 1995, MNRAS (in press) 